\title{
Genetic and Clinical Characterization of Patients with Maturity-Onset of Diabetes of the Young (MODY): Identification of Novel Variations
}

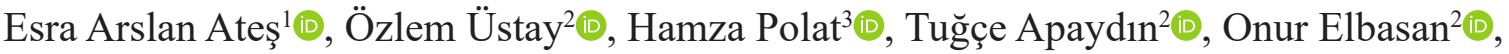 \\ Özlem Y1ldırım ${ }^{4}$, Ahmet İlter Güney ${ }^{3}$ (1)
}

\begin{abstract}
${ }^{1}$ Genetic Diseases Diagnosis Center, Marmara University Pendik Training and Research Hospital, İstanbul, Turkey
${ }^{2}$ Department of Endocrinology and Metabolism, Marmara University School of Medicine, İstanbul, Turkey

${ }^{3}$ Department of Medical Genetics, Marmara University School of Medicine, Istanbul, Turkey

${ }^{4}$ Department of Molecular Biology and Genetics, İstanbul University, İstanbul, Turkey
\end{abstract}

Background: Maturity-onset diabetes of the young (MODY) is a rare monogenic type of diabetes, and accounts for $2-5 \%$ of all diabetes cases. An early age of onset, a family history supporting autosomaldominant inheritance, insulin resistance, and the absence of autoimmunity are the major characteristics of MODY. However, genetic testing is crucial for diagnosis.

Aims: To investigate the 7 MODY-related genes and clinical findings of patients with a preliminary clinical diagnosis of MODY.

Study Design: Retrospective cross-sectional study.

Methods: In this study, 7 genes (KCNJ11, ABCC8, INS, GCK, $H N F 4 A, H N F 1 A$, and $H N F 1 B$ ) related to MODY were screened via targeted sequencing in 182 cases with a confirmed pre-diagnosis of MODY. The clinical characteristics of the patients were evaluated retrospectively.
Results: A total of 182 patients, $48 \%$ of whom were women, between the ages of 18-62 were included in the study. In 30 cases (16.4\%), 28 different pathogenic variations were found, of which 20 were previously reported and 8 were novel variations segregated by disease within the family. Pathogenic variations were detected in the following genes in order of mutation frequency; GCK, HNF1A, ABCC8, HNF4A, HNF1B and KCNJ11. Interestingly, six of the 30 cases $(20 \%)$ carried a pathogenic variation in the ABCC 8 gene. No mutation was detected in the INS gene. A family history of vertically transmitted diabetes and elevated $\mathrm{HbA} 1 \mathrm{C}$ at the time of diagnosis were found in $20(66 \%)$ and 16 (52\%) cases, respectively.

Conclusion: In this series, 28 different pathogenic variations are identified, 8 of which are novel. The rate of pathogenic variation in the $A B C C 8$ gene is unexpectedly high. Two-thirds of cases have a family history of vertically transmitted diabetes.

\section{INTRODUCTION}

Maturity-onset diabetes of the young (MODY), an early age form of adult-type diabetes, is an autosomal-dominant inherited form of monogenic diabetes that occurs due to the dysfunction of the pancreatic beta cells. The characteristic features of MODY are the absence of symptoms of autoimmunity or insulin resistance, preservation of endogenous insulin release, and early-onset diabetes (often before the age of 25). ${ }^{1}$ MODY accounts for approximately $2-5 \%$ of all diabetes cases. As MODY is a genetically heterogeneous group of monogenic diabetes, 14 genes related with MODY have been identified so far. In general, in studies conducted in many different populations, MODY type 3 (21-64\%) and MODY type $2(8-63 \%)$ are the most common types of MODY caused by pathogenic $H N F 1 A$ and $G C K$ gene variations, respectively. ${ }^{2}$ Apart from these, HNF4A, HNF1B, INS, KCNJ11, ABCC8, PDX1, NEUROD1, KLF11, CEL, PAX4, BLK and APPL1 genes have been associated with MODY less frequently. ${ }^{3}$ MODY patients may be misdiagnosed as having type 1 and type 2 diabetes due to clinical heterogeneity. At this point, molecular tests with high sensitivity and specificity are important in determining the correct approach and appropriate treatment.

Corresponding author: Esra Arslan Ateş, Genetic Diseases Diagnosis Center, Marmara University Pendik Training and Research Hospital, Istanbul, Turkey e-mail: esraarslan.md@gmail.com

Received: December 29, 2020 Accepted: May 25, 2021 • DOI: 10.5152/balkanmedj.2021.20155

Available at www.balkanmedicaljournal.org

ORCID iDs of the authors: E.A.A. 0000-0001-5552-8134; Ö.Ü. 0000-0002-7993-955X; H.P. 0000-0003-0833-9968; T.A. 0000-0001-9277-8669; O.E. 0000-0001-8580-9471; Ö.Y. 0000-0003-2674-4828; A.İ.G. 0000-0002-1661-1282.

Cite this article as:

Arslan Ateş E, Üstay Ö, Polat H, et al. Genetic and clinical characterization of patients with maturity-onset of diabetes of the young (MODY): Identification of novel variations. Balkan Med J. 2021;38(5):272-277.

Copyright@Author(s) - Available online at http://balkanmedicaljournal.org/ 
In this study, patients with a preliminary clinical diagnosis of MODY were investigated for 7 MODY-related genes and the genotype-phenotype correlation, with a retrospective evaluation of the accompanying clinical findings.

\section{MATERIAL AND METHODS}

\section{Patient Data}

One hundred eighty-two patients who were referred from the endocrinology department to the medical genetics department with a pre-diagnosis of MODY between 2016 and 2020 were included in this study, which was approved by the Institutional Review Board with the protocol number 09.2020.01. Informed consent was obtained from all patients during personal interviews. The patients were evaluated in terms of their reasons for application, age of onset, and family history. Physical examinations were performed in terms of body mass index, accompanying findings, and vital signs. The patients' blood glucose, cholesterol, autoimmune parameters, and the routine biochemical tests were evaluated retrospectively.

\section{Genetic Tests}

The QIAGEN QIAamp DNA Mini Kit@) (Qiagen, Hilden, Germany) was used for DNA isolation from peripheral blood lymphocytes and the isolation process was performed with the protocol recommended by the kit provider. The workflow of library preparation started with determining DNA quality and quantity. In the next step, all the exons and exon-intron boundaries of $K C N J 11$, $A B C C 8, I N S, G C K, H N F 4 A, H N F 1 A$, and $H N F 1 B$ genes were amplified by multiplex PCRs, using the Multiplicom MODY MasterDx (Agilent, CA) kit. Subsequently, the universal PCR was performed to tag all amplicons with specific molecular identifiers. In the same PCR reaction, all amplicons were also tagged $\mathrm{p} 7$ and $\mathrm{p} 5$ adaptors, which are required for sequencing on the Illumina Miseq platform (Illumina Inc., San Diego, CA). After the universal PCR, the library was purified and the equimolar quantities of each library were pooled. Finally, the pool was denatured by $0.2 \mathrm{~N} \mathrm{NaOH}$ and diluted to $10 \mathrm{pM}$ for loading to the sequencing instrument. The raw data that was generated by the Illumina Miseq in the FASTQ format were analyzed using Sophia DDM (SOPHiA Genetics, Boston). For the confirmation and segregation analysis of the novel variants, the target region was amplified with the designed primers and then sequenced with the ABI Prism 3500 Genetic Analyzer (Thermo Fisher Scientific, MA) device by the Sanger sequencing method. The human reference genome hg19 was used for variant annotation.

\section{Data Analysis}

The data were analyzed using Sophia DDM data analysis software (SOPHiA Genetics, Boston). Coverage was $99.6 \%$ at a minimum depth of 200 reads in 7 MODY-related genes. In order to call variants, the sequencing data was aligned to the human reference genome, hg19. All variations in exons and exon-intron boundaries having minor allele frequency (MAF) under 0.01 in GnomAD were evaluated. ${ }^{4}$ For the pathogenicity evaluation of novel variations, in silico analysis tools were used (Mutation Taster, Deep Neural Network; DANN, Genomic Evolutionary Rate Profiling; GERP). ${ }^{5-7}$
Cases with a clinical diagnosis of diabetes in the proband's family were also screened for segregation analysis. The American College of Medical Genetics (ACMG) guidelines were used for variant classification. $^{8}$

\section{RESULTS}

A total of 182 patients with a pre-diagnosis of MODY, 88 of them (48\%) female and 94 (52\%) male, who were diagnosed with diabetes before the age of 35 and had a family history of diabetes, were included in our study. The patients ranged in age from 18 to 62 years.

In 30 cases $(16.4 \%), 28$ pathogenic variants were found, 20 of which had been reported previously and 8 were novel. (Table 1).

The mean age at diagnosis of the cases with variations was determined as 27 years. Of the 30 cases, $20(66 \%)$ were found to have high blood glucose while being investigated for other reasons, and 7 (23\%) were patients who defined symptoms such as excessive sweating, thirst and fatigue, which are symptoms that suggest diabetes. Three of the cases were detected during family screenings because of their first-degree relatives having been diagnosed with MODY. A history of diabetes showing vertical inheritance in the family was present in 20 cases $(66 \%)$. HbA1C elevation was detected in $16(53 \%)$ of the cases at diagnosis, and $\mathrm{HbA} 1 \mathrm{C}$ was found to be elevated in only $1(10 \%)$ of 10 cases with pathogenic $G C K$ variation and in $6(66 \%)$ of 9 cases with a pathogenic $H N F 1 A$ variation. The clinical characteristics of the cases with pathogenic/ likely pathogenic variations and their distribution according to the related gene are presented in Table 2.

In the study, 10 different pathogenic/likely pathogenic $G C K$ variations were detected in 10 (33\%) of 30 cases, 3 of which were novel (c.287A >C; p.Glu96Ala, c.1246C > T; p.His416Tyr and c.852delC; p.Gly285Valfs $9 *$ ). Of these, 7 variations were missense, 1 was nonsense, and 2 were single-nucleotide deletions causing a shift in the reading frame.

The HNF1A gene was the second most common gene to have pathogenic/likely pathogenic variations, and 8 distinct pathogenic/likely pathogenic variations were detected in 9 cases (30\%), 2 of which were novel (c.375_377del; p.Gln125del and c.1136dupC; p.Val380Cysfs*39). The genes $A B C C 8$ (6 cases), $H N F 4 A$ ( 2 cases), HNF1B ( 2 cases), and $K C N J 11$ (1 case) followed respectively. No mutation was detected in the INS gene in any of the cases. The distribution of mutations according to genes is presented in Figure 1.

We were able to perform a segregation analysis for 8 out of the 16 variations that were not previously classified for pathogenicity. A total of 28 first-degree relatives of 8 cases were screened, and 7 of the variations were shown to be segregating with the disease in the family. For the HNF1A c.1136dupC (p.Val380Cysfs*39) variation, segregation analysis was not possible. However, the variation was considered as likely pathogenic due to the low MAF (not found in GnomAD) and predicted to cause truncated protein. Novel variations were also evaluated using in silico analysis 
TABLE 1. Pathogenic and Likely Pathogenic Mutations Detected in the Study

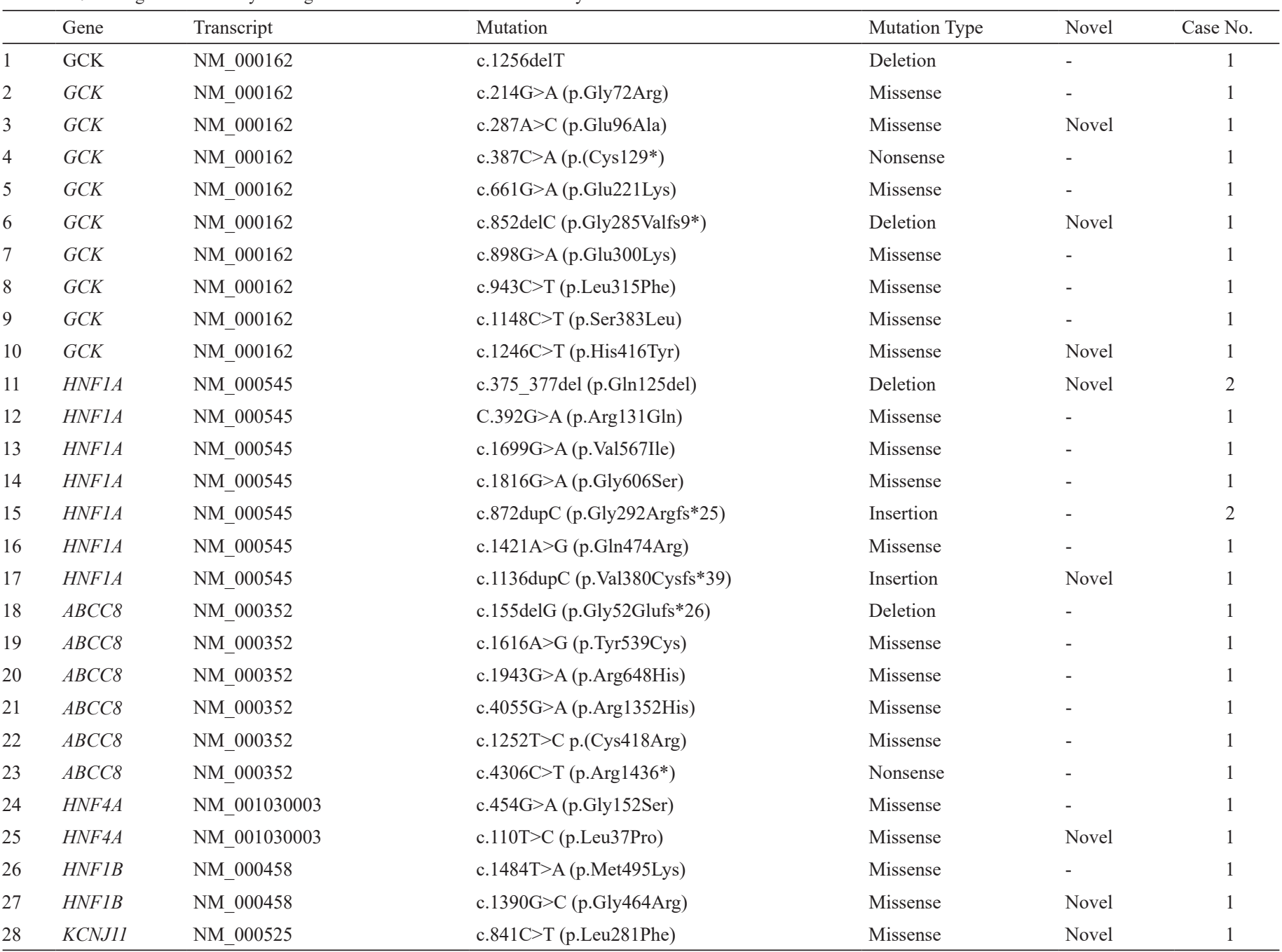

programs and classified as likely pathogenic, and have been summarized in Table 3 with pathogenicity criteria.

The parents of the case (26-year-old, female) carrying the GCK c. $45+4 \mathrm{~T}>\mathrm{A}$ variation both had been diagnosed with diabetes. The variation was inherited from the 49-year-old mother. No other family member was evaluated therefore the variation was classified as a "variant of uncertain significance" (VUS) (Table 4).

The other 7 variations for which we were not able to screen the related family members, were classified as VUS according to the ACMG criteria, and are presented in Table 4.

\section{DISCUSSION}

In this study, we present the outcomes of a systematic search for 7 MODY-related gene mutations in a Turkish MODY cohort. In 30 cases $(16.4 \%)$, a pathogenic variant was detected in 1 of these 7 genes. Totally, 8 of these mutations were novel, of which 3 were in the GCK gene, 2 in the HNF1A gene, 1 in the HNF4A gene,
1 in the HNF1B gene, 1 in KCNJ11 gene. Similar to our study, in a previous study including 106 cases, $17 \%$ of the cases had a pathogenic variant in 1 of these 7 genes. ${ }^{9}$ The pathogenic variation frequency was $29 \%$ in 42 children diagnosed with MODY. However, in this study, 5 genes were common with our study (GCK, HNF1A, HNF4A, HNF1B, and INS), and an additional 6 genes (PDX1, NEUROD1, KLF11, CEL, PAX4, and BLK) were also screened. The difference between this study and our study may be due to the presence of the additional genes, as well as the screening of distinct age groups.

In the literature, 14 genes (HNF4A, GCK, HNF1A, PDX1, HNF1B, NEUROD1, KLF11, CEL, PAX4, INS, BLK, ABCC8, KCNJ11, and $A P P L 1$ ) have been associated with MODY, and pathogenic $G C K$ and $H N F 1 A$ variations are detected in approximately half of the MODY patients. ${ }^{3}$ Among the cases where we detected mutations in this study, our detection rates for mutation in the $G C K$ and $H N F 1 A$ genes were $33 \%$ and $30 \%$, respectively, consistent with the literature. 
TABLE 2. Clinical Characteristics of Cases with Mutations in the Screened MODY Genes

\begin{tabular}{|c|c|c|c|c|c|c|c|}
\hline & $\begin{array}{c}\text { Mutation ( }+ \text { ) Patients } \\
\qquad(n=30)\end{array}$ & $\operatorname{GCK}(n=10)$ & $\operatorname{HNF} 1 \mathrm{~A}(n=9)$ & $\begin{array}{c}\mathrm{ABCC} 8 \\
(n=6)\end{array}$ & HNF4A $(n=2)$ & HNF1B $(n=2)$ & $\begin{array}{c}\mathrm{KCNJ} 11 \\
(n=1)\end{array}$ \\
\hline \multicolumn{8}{|l|}{ Referral reason } \\
\hline Incidental high blood glucose & $20(67 \%)$ & $7(70 \%)$ & $5(55 \%)$ & $4(67 \%)$ & 2 & 1 & 1 \\
\hline Diabetes-specific complaints & $7(22 \%)$ & $1(20 \%)$ & $4(45 \%)$ & $2(33 \%)$ & - & - & - \\
\hline Family screening & $3(11 \%)$ & 1 & 1 & - & - & 1 & - \\
\hline Age of onset (min-max) & $12-49$ & $26-49$ & $12-29$ & $24-45$ & $34-43$ & $15-32$ & 24 \\
\hline BMI (min-max), mg/dL & $20-44$ & $20-28$ & $23-28$ & $21-41$ & $24-30$ & $28-31$ & 32 \\
\hline FBG (min-max) & $96-487$ & $121-145$ & $101-160$ & $96-142$ & $105-487$ & $121-195$ & 111 \\
\hline \multicolumn{8}{|l|}{$\mathrm{HbA1C}$} \\
\hline$<7 \%$ & $16(53 \%)$ & $9(90 \%)$ & $3(33 \%)$ & $3(50 \%)$ & - & - & 1 \\
\hline$>7 \%$ & $14(46,6 \%)$ & 1 & $6(67 \%)$ & $3(50 \%)$ & 2 & 2 & - \\
\hline Hyperlipidemia & $6(19 \%)$ & $3(30 \%)$ & - & $2(33 \%)$ & - & - & 1 \\
\hline Nephropathy & $6(19 \%)$ & $2(20 \%)$ & - & 1 & 1 & 1 & 1 \\
\hline Neuropathy & $3(10 \%)$ & $2(20 \%)$ & - & 1 & - & - & - \\
\hline Hepatosteatosis & $8(25 \%)$ & $3(30 \%)$ & - & $3(50 \%)$ & - & 1 & 1 \\
\hline
\end{tabular}

BMI, body mass index; FBG, fasting blood glucose.

The GCK (OMIM *138079) gene, localized on chromosome 7 p13 and spanning 10 exons, encodes the glucokinase enzyme responsible for glucose phosphorylation, the first step of glycolysis. More than 900 mutations have been listed for the GCK gene, in the Human Gene Mutation Database Professional (HGMD), 2020. ${ }^{9,10}$ There is no hotspot region in which mutations cluster in the gene. The majority of pathogenic variations (70\%) are missense and nonsense variations. ${ }^{11}$ In our study, in 10 unrelated cases, 10 distinct $G C K$ variations spreading over the whole gene were detected. Altogether, 7 (70\%) of the pathogenic variations were missense, 2 were single-nucleotide deletions and 1 was a nonsense variation. Three of these variations have been detected for the first time in this study. Pathogenic variations in the $G C K$ gene cause MODY type 2 (OMIM \# 125851), characterized by mild diabetes

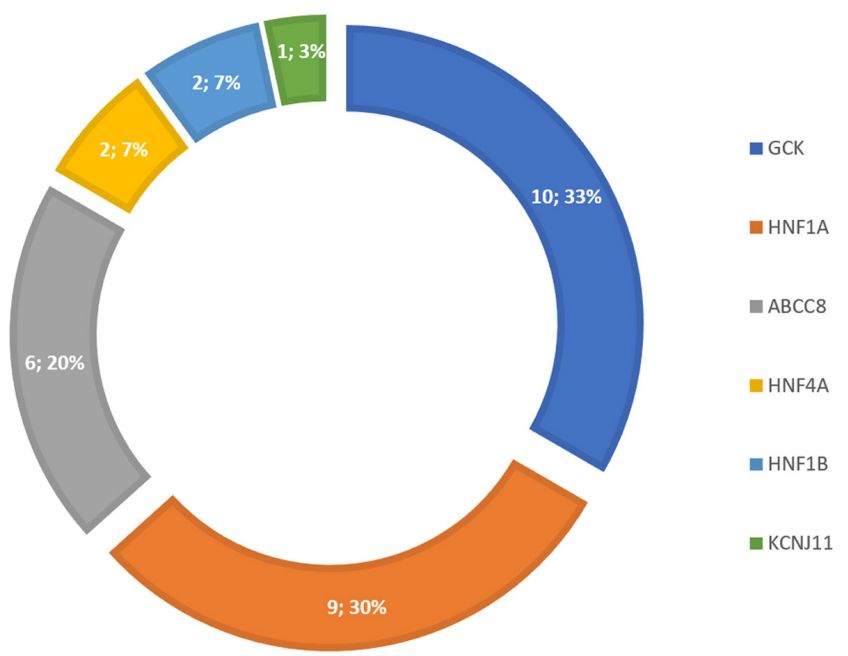

FIG. 1. Mutation detection rate in the screened genes. that does not require treatment. ${ }^{1}$ In addition, the risk of microvascular and macrovascular complications in these cases was similar to controls without diabetes. ${ }^{12}$ In 9 out of the 10 GCK mutation carriers, the blood glucose levels were found to be moderately elevated, and the $H b A 1 C$ levels were below $7 \%$. On the other hand, 2 patients had retinopathy and 2 patients had neuropathy. However, due to the low number of cases, a statistical interpretation was not considered possible.

In our study, the $H N F 1 A$ was the second most frequent gene in which a pathogenic variation was detected, accounting for $30 \%$ of all cases of pathogenic variations detected. The HNF1A gene is localized on chromosome $12 \mathrm{q} 24$, spans 10 exons, and encodes a tissue-specific transcription factor that is important in maintaining the functions of differentiated pancreatic islet cells. ${ }^{13}$ More than 500 pathogenic $H N F 1 A$ variations, mostly missense and nonsense, have been identified in the HGMD database. ${ }^{10}$ Of the 7 different HNF $1 A$ mutations detected in 9 cases in our study, 4 of them were missense, 2 of them were insertion, and 1 of them was deletion, as 2 of them caused a frameshift. Two of these variations were novel. The c.872dupC (p.Gly292Argfs*25) variation detected in HNF1A was previously defined, and is one of the recurrent pathogenic variations detected in 2 unrelated cases in the study. The novel $H N F 1 A$ c.375_377del (p.Gln125del) variation was also detected in 2 unrelated cases in this study. HNF1A mutations cause MODY type 3 . Hyperglycemia is detected at an earlier age in asymptomatic cases or cases with mild osmotic findings. The risk of microvascular and macrovascular complications depends on blood glucose regulation. ${ }^{3}$ In our study, pathogenic $H N F 1 A$ variation carriers generated the group of cases diagnosed at the earliest age, and the youngest patient diagnosed was 12 years old. An asymptomatic 29-year old patient was the oldest to be diagnosed in this group. None of the patients had vascular complications at the time of the study. 

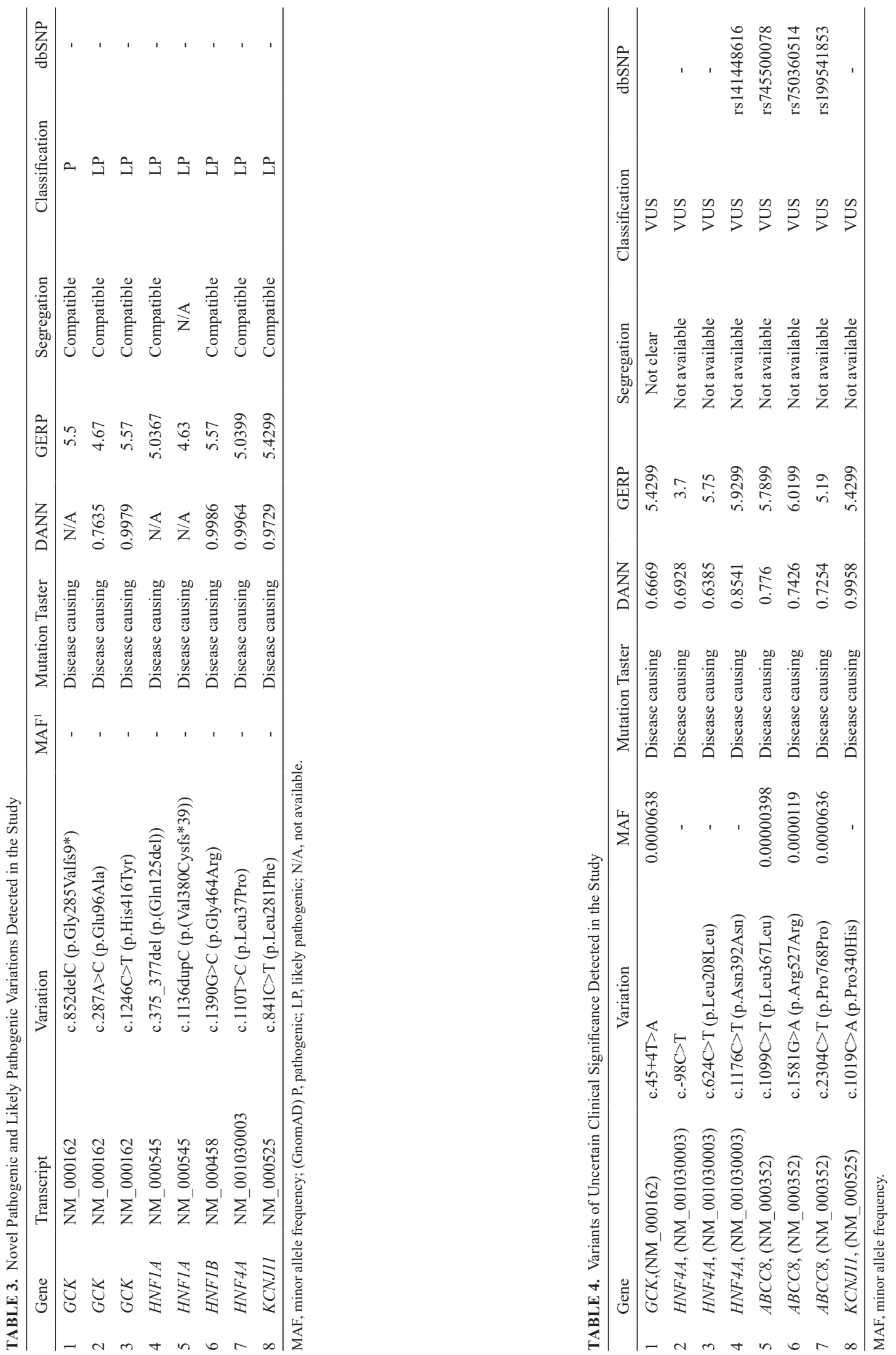
In our cohort, pathogenic $A B C C 8$ variations were found in $20 \%$ of all pathogenic variation carriers, as the third most frequently affected gene. The $A B C C 8$ gene, which is responsible for at least $1 \%$ of MODY cases in the literature, encodes the sulfonylurea receptor. Since biallelic pathogenic variations of this gene cause congenital hyperinsulinemic hypoglycemia (CHI), genetic counseling of the patients is of particular importance. ${ }^{3,14}$ There are about 700 mutations of the $A B C C 8$ gene in the HGMD. There is a wide spectrum of variations, more than half of which are missense and nonsense variations. ${ }^{10}$ Four missens, 1 nonsense and one insertion causing frameshift variation were detected, which of all were previously reported as pathogenic variation.

In addition, in our study, a total of 3 novel likely pathogenic variations were found in the HNF1B, HNF4A and KCNJ11 genes, and no variation was found in the INS gene suggested to be pathogenic.

In conclusion, in this study, 7 genes associated with MODY (KCNJ11, ABCC8, INS, GCK, HNF4A, HNF1A, and HNF1B) were screened via targeted next-generation sequencing (NGS) in 182 cases evaluated with a pre-diagnosis of MODY, and the molecular etiology was elucidated in $30(16.4 \%)$ of them. The molecular etiology could not be revealed in $83.6 \%$ of the cases. However, variations in the regulatory sequences in the deep intronic and untranslated regions of these genes and large copy number changes could not be excluded in these patients due to the limitation of the method. In addition, the second limitation of our study was that, we were able to screen only 7 genes, although, to date, 14 genes were found to be associated with MODY. It will be possible to elucidate the etiology by screening these cases with panels containing other genes associated with MODY, and more comprehensive methods such as exome or genome sequencing may be necessary for genes that have not yet been related with MODY.

Ethics Committee Approval: Ethical committee approval was received from the Ethics Committee of Marmara University School of Medicine, (Approval No: 09.2020.01).

Patient Consent for Publication: Informed consent was obtained from all patients during personal interviews.
Data-sharing Statement: The data that support the findings of this study are available from the corresponding author upon reasonable request.

Author Contributions: Concept - E.A.A., Ö.Ü.; Design - E.A.A., Ö.Ü.; Supervision A.I.G.; Materials - T.A., Ö.Y.; Data Collection and/or Processing - E.A.A., H.P., T.A., O.E., Ö.Y.; Analysis and/or Interpretation - E.A.A., Ö.Ü.; Literature Review - H.P., T.A.; Writing - E.A.A.; Critical Review - E.A.A., Ö.Ü., H.P., T.A., O.E., Ö.Y., A.İ.G.

Conflict of Interest: The authors have no conflicts of interest to declare.

Funding: The authors declared that this study has received no financial support.

\section{REFERENCES}

1. Anık A, Çatlı G, Abacı A, Böber E. Maturity-onset diabetes of the young (MODY): an update. J Pediatr Endocrinol Metab. 2015;28(3-4):251-263. [CrossRef]

2. Giuffrida FM, Reis AF. Genetic and clinical characteristics of maturity-onset diabetes of the young. Diabetes Obes Metab. 2005;7(4):318-326. [CrossRef]

3. Peixoto-Barbosa R, Reis AF, Giuffrida FMA. Update on clinical screening of maturity-onset diabetes of the young (MODY). Diabetol Metab Syndr. 2020;12:50. [CrossRef]

4. Karczewski KJ, Francioli LC, Tiao G, et al. The mutational constraint spectrum quantified from variation in 141,456 humans. Nature. 2020;581(7809):434-443. [CrossRef]

5. Schwarz JM, Cooper DN, Schuelke M, Seelow D. MutationTaster2: mutation prediction for the deep-sequencing age. Nat Methods. 2014;11(4):361-362. [CrossRef]

6. Quang D, Chen Y, Xie X. DANN: a deep learning approach for annotating the pathogenicity of genetic variants. Bioinformatics. 2015;31(5):761-763. [CrossRef]

7. Cooper GM, Stone EA, Asimenos G, et al. Distribution and intensity of constraint in mammalian genomic sequence. Genome Res. 2005;15(7):901-913. [CrossRef]

8. Richards S, Aziz N, Bale S, et al. Standards and guidelines for the interpretation of sequence variants: a joint consensus recommendation of the American College of Medical Genetics and Genomics and the Association for Molecular Pathology. Genet Med. 2015;17(5):405-424. [CrossRef]

9. Iynedjian PB. Mammalian glucokinase and its gene. Biochem J. 1993;293( 1):1-13 [CrossRef]

10. Stenson PD, Mort M, Ball EV, et al. The human gene mutation database: 2008 update. Genome Med. 2009;1(1):13. [CrossRef]

11. Aykut A, Karaca E, Onay H, et al. Analysis of the GCK gene in 79 MODY type 2 patients: a multicenter Turkish study, mutation profile and description of twenty novel mutations. Gene. 2018;641:186-189. [CrossRef]

12. Steele AM, Shields BM, Wensley KJ, et al. Prevalence of vascular complications among patients with glucokinase mutations and prolonged, mild hyperglycemia. JAMA. 2014;311(3):279-286. [CrossRef]

13. Valkovicova T, Skopkova M, Stanik J, Gasperikova D. Novel insights into genetics and clinics of the HNF1A-MODY. Endocr Regul. 2019;53(2):110-134. [CrossRef]

14. Bowman P, Flanagan SE, Edghill EL, et al. Heterozygous ABCC8 mutations are a cause of MODY. Diabetologia. 2012;55(1):123-127. [CrossRef] 